The Astrophysical JouRnAL, 558:309-322, 2001 September 1

(C) 2001. The American Astronomical Society. All rights reserved. Printed in U.S.A.

\title{
INTRINSIC COLORS OF STARS IN THE NEAR-INFRARED
}

\author{
Jorge R. Ducati, Claudio M. Bevilacqua, Sandro B. Rembold, and Daiana Ribeiro \\ Departamento de Astronomia, Universidade Federal do Rio Grande do Sul, Porto Alegre, Brazil; ducati@if.ufrgs.br; erex@if.ufrgs.br; rembold@if.ufrgs.br; \\ claiana@if.ufrgs.br \\ Received 1999 November 4; accepted 2001 May 3
}

\begin{abstract}
Intrinsic infrared colors of stars in the Johnson 11 color system are derived. The database is a list of 3946 stars with observations in UBVRIJHKLMN, of all spectral types and luminosity classes, including carbon, T Tauri, and Wolf-Rayet stars. Intrinsic colors were derived from the zero-reddening curve that can be defined in the bluer side of temperature versus observed color diagrams. In a sample of stars of the same spectral type, the bluest stars are considered to have an observed color very near their intrinsic color. The comparison with former derivations from Johnson and Koornneef presents significant differences: new $M$ and $N$ colors, for all spectral types, are bluer than published values, the differences being more important for giants and supergiants; the amplitude of color values in these new results is wider.
\end{abstract}

Subject headings: infrared: stars - stars: fundamental parameters - techniques: photometric

\section{INTRODUCTION}

The intrinsic colors of stars have been derived mainly for the visible part of the spectrum (Schmidt-Kaler 1982 for $U B V$; Philip \& Egret 1980 for uvby). In the infrared the first derivation was that of Johnson (1966), followed by those of Lee (1970), Frogel et al. (1978), Whittet \& van Breda (1980), Koornneef (1983b), Bessell \& Brett (1988), Bouchet, Manfroid, \& Schmider (1991), and Wegner (1994). Johnson's pioneering work was based on the assumption that there is no interstellar reddening out to distances of up to $100 \mathrm{pc}$ from the Sun. The intrinsic colors of dwarfs and giants were the average of the observed colors, separated by spectral type; for supergiants he used a method similar to that of Kron (1958), which introduced corrections for interstellar reddening. Johnson's tables are still the most widely used, in spite of results obtained much later. Results from Koornneef (1983a, 1983b) were derived using blackbody models and do not differ significantly from Johnson's. Other works, even though representing improvements for certain classes of stars, do not cover all spectral types and are based on samples of limited size. Since the compilation of the catalog of Ducati (1993), a much larger amount of data has become available, allowing direct, statistically significant derivation of infrared intrinsic colors. In this paper, we first discuss the quality of our database in terms of homogeneity of published observations and validity of averaging. Next, we present the method, discussing its limitations. Finally, results are discussed and compared with earlier derivations.

\section{DATA}

The NASA infrared catalogs (CIO catalogs; Gezari et al. 1993), obtained from both ground and space observations, are the most comprehensive set of near- to far-infrared data on astronomical objects. Despite its near completeness in terms of literature search, the catalog lacks some additional information that would make it more useful, such as spectral types, $U B V R I$ photometry, and galactic coordinates. In addition, the same object frequently has several identifications or even differences in coordinates. Using the CIO catalogs as sources for the near-infrared data, a catalog of UBVRIJHKLMN photometry of stars was compiled by
Ducati (1993), containing infrared and subsidiary information for 3946 objects. Extensive information on how this catalog was compiled is available (Ducati 1993), but the main constraints were as follows: the bulk of $J$ to $N$ data came from Gezari et al. (1993) and its updates; this comprises observational data from hundreds of references, which makes a case-by-case study of the observational, instrumental, and reducing techniques used in each paper very difficult and almost prevents an accurate standardization. Our first step was to fix narrow limits in the $J H K L M N$ passbands, consistent with those defined by Johnson (1966). Attention was given to the beamwidth used in the observations, information given in the CIO catalogs. This is important because many observations concern stars embedded in nebulae (viz., in the region of Orion Nebula), implying that the field around the star also radiates in the infrared; different apertures give different contributions from the nebula to the measured infrared magnitude. In these cases we discarded all observations except those with the smallest diaphragm.

Even with these filters, our preliminary sample included many stars with multiple observations; averaging procedures are described in $\S 2.1$. This does not concern the $R$ and $I$ colors in the Johnson system, which were extracted from the lists of Lanz (1986), Hoffleit \& Jaschek (1982), and Hoffleit, Saladyga, \& Wlasuk (1983) and from the SIMBAD database and for which no averaging was done. Identifications of carbon and T Tauri stars were made possible by the catalogs in Lang (1992), the infrared data coming from Gezari et al. (1993).

\subsection{Averaging Procedures}

We begin with a general consideration: the original purpose of compiling a catalog is to form a database that is larger than any of the many sources used. In merging data from several sources, it is unavoidable and in fact desirable that stars common to more than one source exist. The data from these common stars must be averaged, if possible; in fact, a catalog will be useful if averaging is indeed possible, because then all the data will be expressed in the same system. With this philosophy in mind, we took the steps described below. 
The fact that many stars have been observed several times implies that, if some averaging has to be made, preliminary testing has to legitimate it. Averaging astronomical results from different observers can only be done after careful verification of data homogeneity, including whether the published results were reduced to the same photometric system. This problem was approached by comparing published infrared observations of the same stars made by different authors. Many papers did not mention whether their published observational data were reduced to some standard system, such as that of Johnson. As a first verification, we selected a sample of stars for which multiple observations from many papers were available. An inspection showed that the photometric indices $J$ to $N$ were in general very close. This strongly suggests that the papers in general contained data already reduced to the Johnson system, because if those papers had published their observations as instrumental magnitudes, or even in some other photo-

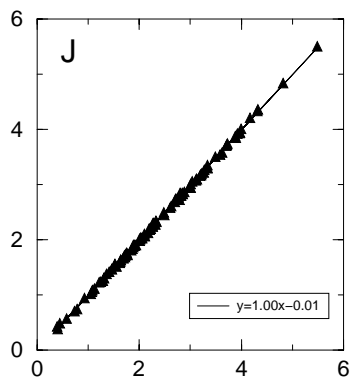

(a)

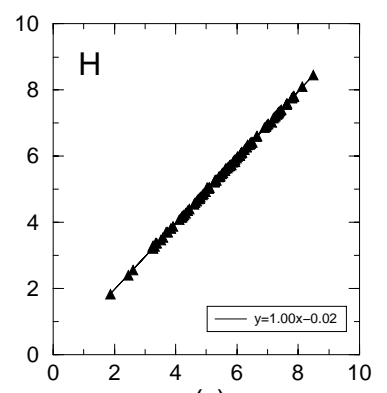

(c)

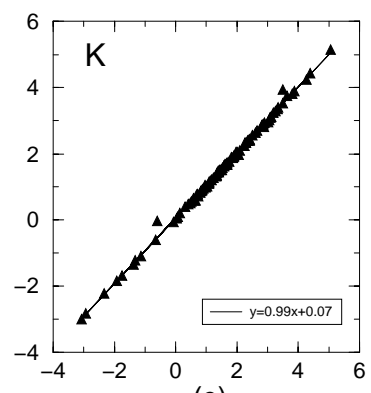

(e)

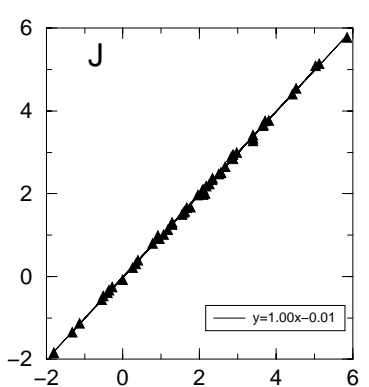

(b)

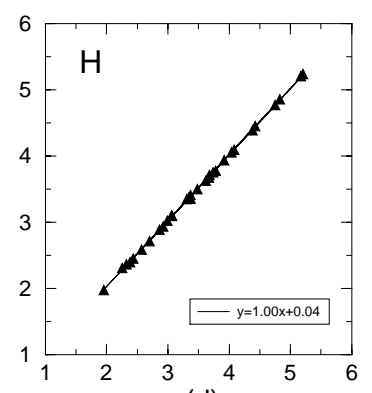

(d)

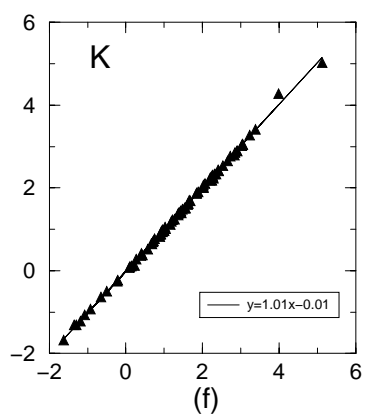

(f)

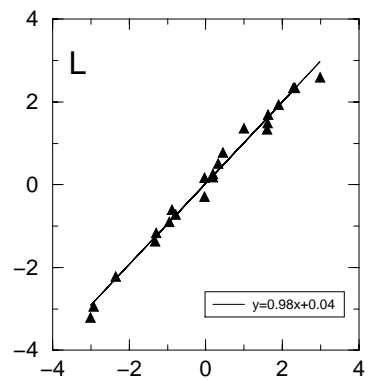

(a)

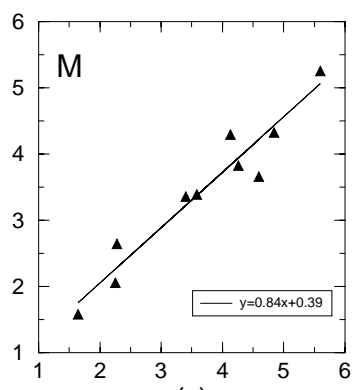

(c)

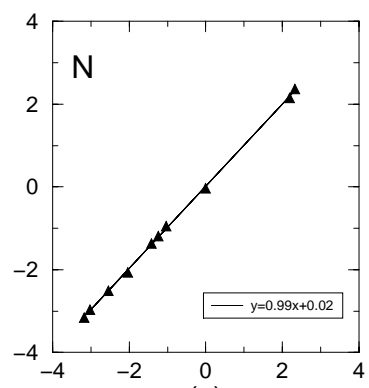

(e)

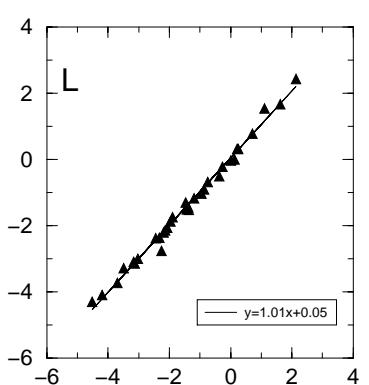

(b)

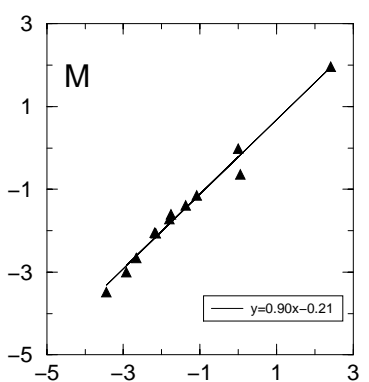

(d)

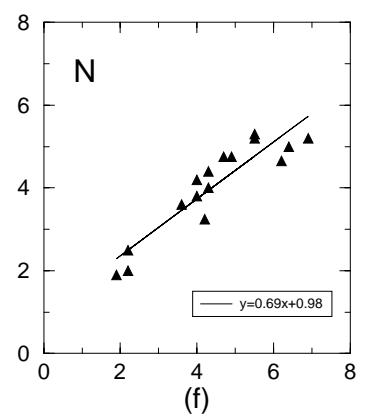

FIG. 1.-Correlation of data for (a) Carter (1990) vs. Glass (1974), (b) Engels et al. (1981) vs. Johnson et al. (1966), (c) Thé, Wesselius, \& Janssen (1986) vs. Whittet \& van Breda (1980), (d) Carter (1990) vs. Allen \& Cragg (1983), (e) Selby et al. (1988) vs. Johnson et al. (1966), and ( $f$ ) Glass (1974) vs. Johnson et al. (1966). metric system, the closeness we found in this first comparion would not have occurred.

possibility of making averages, we took those papers which more significantly to the compilation of the two papers and for the colors JHKLMN, the photometric indices for the common stars. The comparisons, expressed correlation parameters, give an indication on how far system. Figures 1 and 2 illustrate some of these correlations. We see that the correlations, besides being linear, in most very small dispersion. This clearly indicates Johnson system. Differences between papers tend to fall within observational errors. We conclude that it is valid to average observations of every color for each star with several observations. For example, in Figure $1 a$, we show
FIG. 2.-Correlation of data for (a) Catchpole et al. (1979) vs. Gillett, Merrill, \& Stein (1971), (b) Gehrz \& Woolf (1971) vs. Johnson et al. (1966), (c) Thé et al. (1986) vs. Gehrz, Hackwell, \& Jones (1974), (d) Sinton \& Tittemore (1984) vs. Gehrz \& Woolf (1971), (e) Tokunaga (1984) vs. Gehrz et al. (1974), and ( $f$ ) Rydgren, Strom, \& Strom (1976) vs. Cohen (1974). 
the comparison between the $J$ color of Carter (1990) and Glass (1974), for which we found 119 common stars. The correlation is very close to unity, and the dispersion of data is of the order of photometric errors. This shows that both papers deal with data expressed in photometric systems which are very close, making averaging possible. Information supplied in Figures 1 and 2 and its captions support these conclusions. Obviously, many pairs of papers have a $100 \%$ correlation, since they are part of series of publications from the same research group, using the same instruments and reduction methods. This makes merging a direct operation.

\subsection{Expected Errors in JHKLMN Photometry}

An important question concerns the error associated with a near-infrared observation. We know that $U B V$ magnitudes have an internal error of about 0.01 to $0.02 \mathrm{mag}$. We analyzed our data looking for stars whose final infrared colors were the result of averaging. The standard deviations of these averages were also averaged for each of the $J H K L M N$ colors and for spectral types divided into $\mathrm{OB}$, $\mathrm{AF}$, and GKM groups of normal stars, as well as for WolfRayet, carbon, and T Tauri stars. Table 1 shows these averaged standard deviations. The distribution of the standard deviations for color $J$ is shown in Figure 3 to be strongly concentrated toward values near zero. Sterken $\&$ de Loore (1982) have calculated expected photometric errors for $J H K L M$ of Wolf-Rayet stars, finding standard deviations of the observed colors smaller than our values. Carter (1990) estimates an accuracy of $0.02 \mathrm{mag}$ for $J, H$, and $K$ and 0.05 mag for $L$, independent of spectral types.

The results in Table 1 show a clear trend, in the sense that the precision of infrared photometry is better for shorter wavelengths. For example, $J$ photometry has an accuracy (for OB stars) comparable to $U B V$; precision deteriorates toward $N$ and for later spectral types. This is expected, because it is well known that photometry toward $10 \mu \mathrm{m}$ meets instrumental difficulties. Moreover, diversity of luminosity classes and evolutionary stages for late stars tends to increase the dispersion for those classes. We note that the size of each subsample is not relevant to the dispersion value. For example, $K$ photometry provides data for 699 stars in the GKM group. Nevertheless, the dispersion is greater for those stars than that for $\mathrm{OB}$ and $\mathrm{AF}$ stars, derived from smaller samples. Also, we note that the great-

TABLE 1

ERRORS IN JHKLMN PHOTOMETRY

\begin{tabular}{ccccccc}
\hline \hline Star Type & $J$ & $H$ & $K$ & $L$ & $M$ & $N$ \\
\hline O, B ....... & 0.045 & 0.046 & 0.061 & 0.062 & 0.115 & 0.105 \\
& 387 & 359 & 464 & 351 & 129 & 38 \\
A, F ....... & 0.033 & 0.032 & 0.036 & 0.064 & 0.134 & 0.123 \\
& 273 & 201 & 315 & 154 & 57 & 18 \\
G, K, M ... & 0.058 & 0.062 & 0.063 & 0.079 & 0.110 & 0.187 \\
& 624 & 485 & 699 & 427 & 137 & 62 \\
WN, WC ... & 0.070 & 0.068 & 0.079 & 0.116 & 0.121 & 0.158 \\
& 26 & 33 & 51 & 27 & 23 & 12 \\
Carbon ..... & 0.211 & 0.166 & 0.162 & 0.130 & 0.159 & 0.298 \\
& 87 & 82 & 91 & 61 & 27 & 6 \\
T Tauri .... & 0.156 & 0.167 & 0.150 & 0.248 & 0.464 & 0.252 \\
& 27 & 42 & 40 & 59 & 19 & 2 \\
\hline
\end{tabular}

NoTE.-For each type, the first row shows the mean standard deviation $\bar{\sigma}$ and the second shows the number of stars.

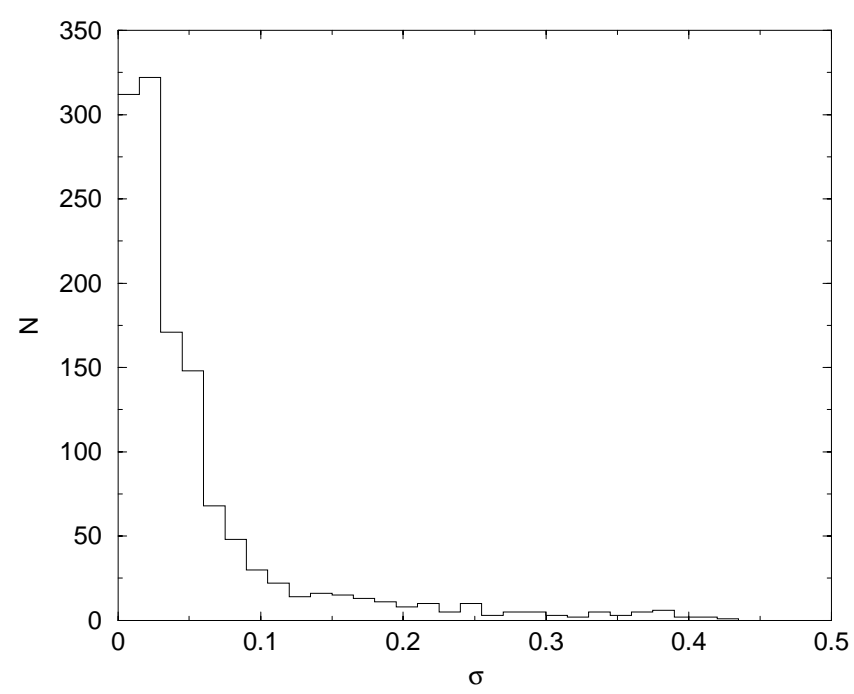

FIG. 3.-Histogram of standard deviations of $J$ color

est dispersion, 0.187 in $N$ photometry for GKM stars, was derived from 62 stars; however, this is greater than the dispersion in $M$ for AF stars, which was derived from fewer stars $(\bar{\sigma}=0.134$, from 57 stars $)$.

\section{DERIVATION OF INTRINSIC COLORS}

We are dealing with a $V-\lambda$ catalog. Given the rather large amount of data available, it is possible to make a direct approach to the problem of deriving intrinsic colors. We have several hundred points corresponding to stars, with a large range of distances and to a large range of reddenings. It is therefore natural that the nearest stars will show little, if any, reddening. Given a group of stars with the same spectral type and luminosity class, those with the smallest $V-\lambda$ tend to have a near-zero reddening. For a range of spectral types, it is possible to draw an envelope within which reddening is close to zero, giving the intrinsic color of each spectral type. Diagrams of temperature-color indices were constructed for the RIJHKLMN colors, for each spectral type, and for luminosity classes V, III, and I; for Wolf-Rayet, carbon, and T Tauri stars we used spectral classes instead of temperature. Temperatures for normal stars were taken from Allen (1973), where a fit was made to provide information for intermediate spectral types. For the other three classes, the spectral sequences as given by Jaschek \& Jaschek (1987) and Kaler (1989) were used. Next, we investigated the question of the size of the gap between the least reddened stars and the envelope that is the locus of zero reddening. This question can be addressed by considerations about the mean distance between stars of the same luminosity class. Densities of various star populations, as given by Mihalas \& Binney (1981), can be used to show that, in this region of the Galaxy, the mean distance between dwarfs is about $2.1 \mathrm{pc}$ and that, for giants and supergiants, it is about 14.3 pc. Given that those distances are small for reddening effects and that our sample tends to be complete toward brighter and less reddened stars, we took the envelope line as the zero-reddening curve, corresponding to the intrinsic colors. The final envelopes were drawn from best-fit polynomials or more complex functions; from A to colder stars the temperature scale was expanded, because the correlation between temperature and color indices becomes steeper. 


\section{RESULTS}

Diagrams of temperature-color indices are shown in Figures 4, 5, 6, and 7. Figures 8, 9, and 10 present spectral class-color indices diagrams for Wolf-Rayet, $\mathrm{T}$ Tauri, and carbon stars.
For these stars, in general, only upper limits can be derived from the data; actual intrinsic colors are probably bluer than the bluest stars in all classes. For T Tauri stars, we note that the scatter in the colors is smaller for late spectral types; this is expected, because late types are less
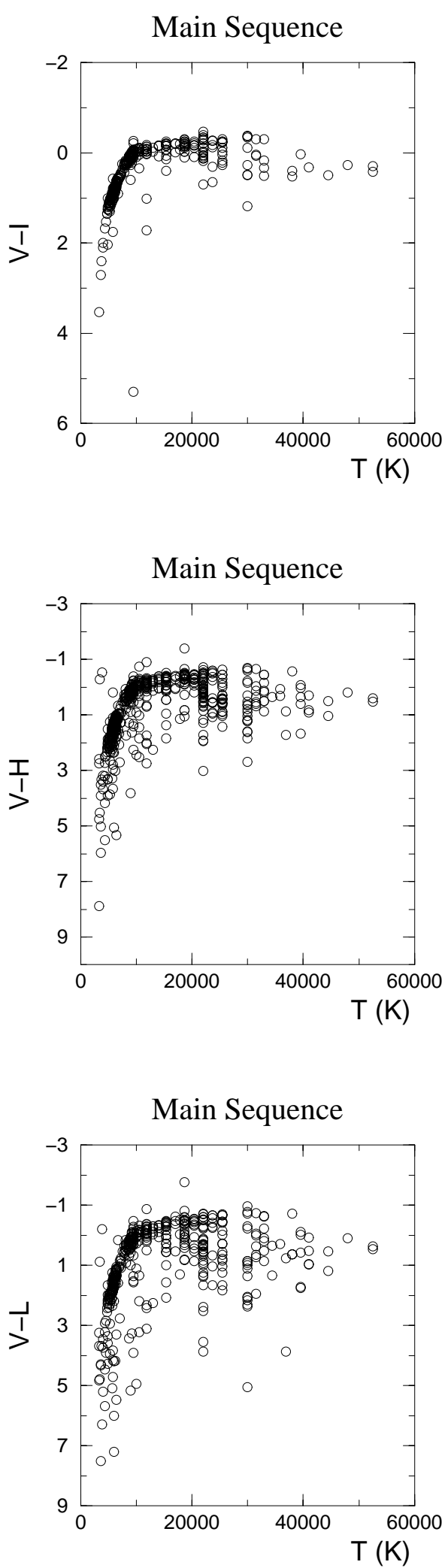

FIG. 4.-Temperature-color indices for main-sequence stars 

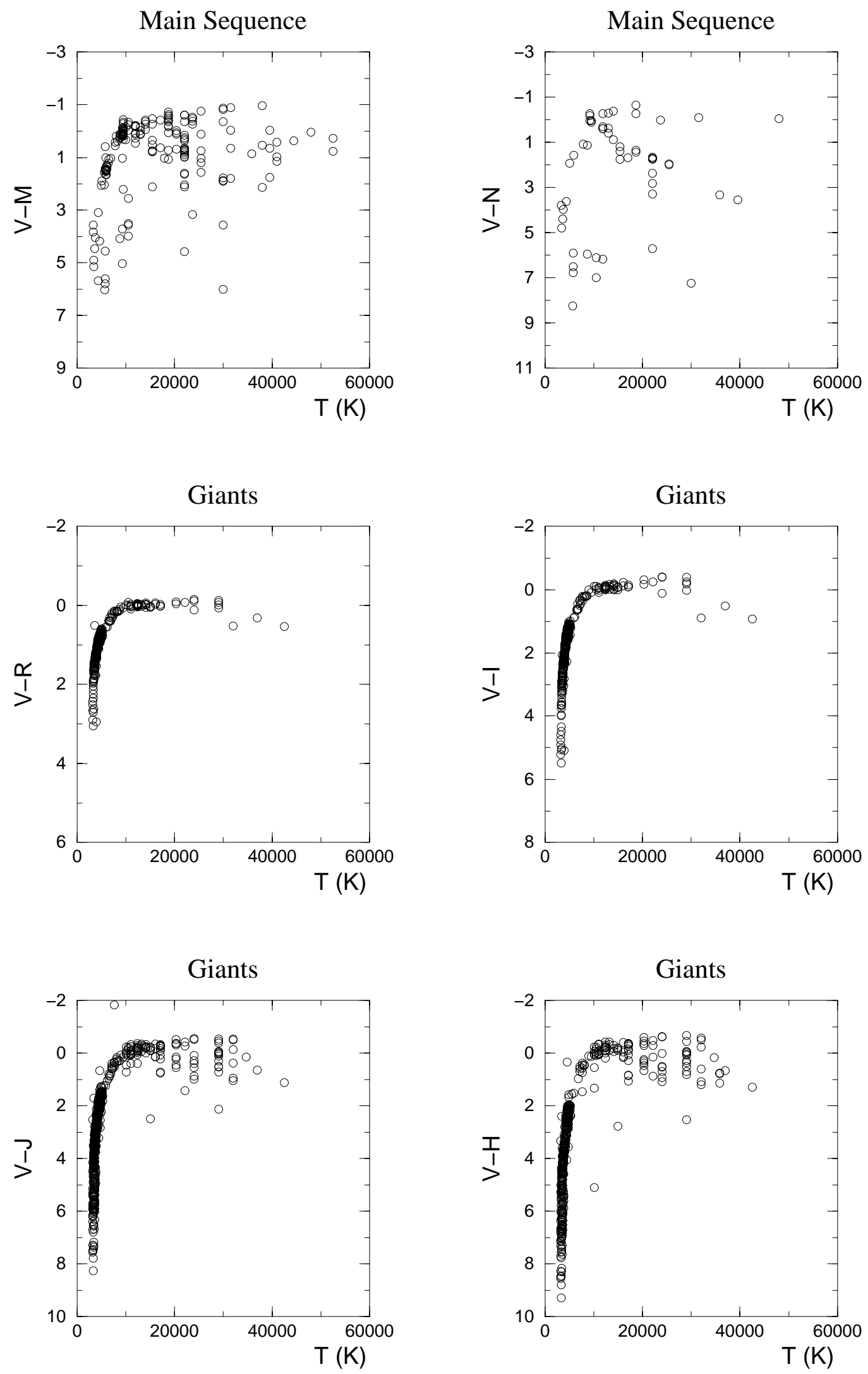

FIG. 5.-Same as Fig. 4, but for main-sequence and giant stars 
Giants

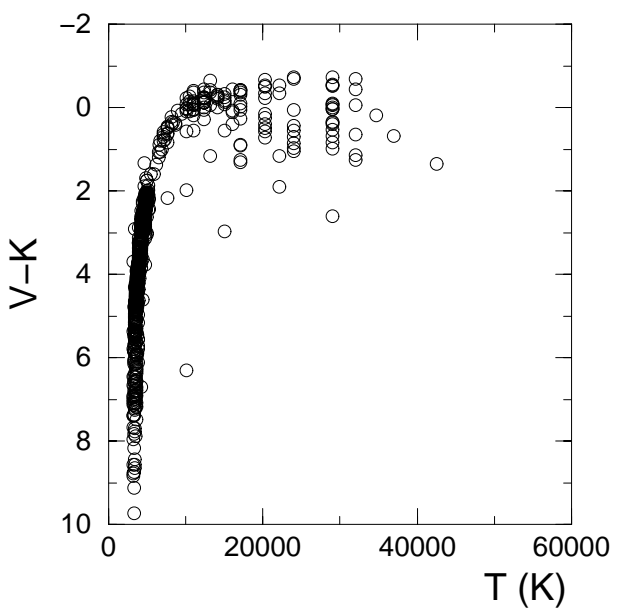

Giants

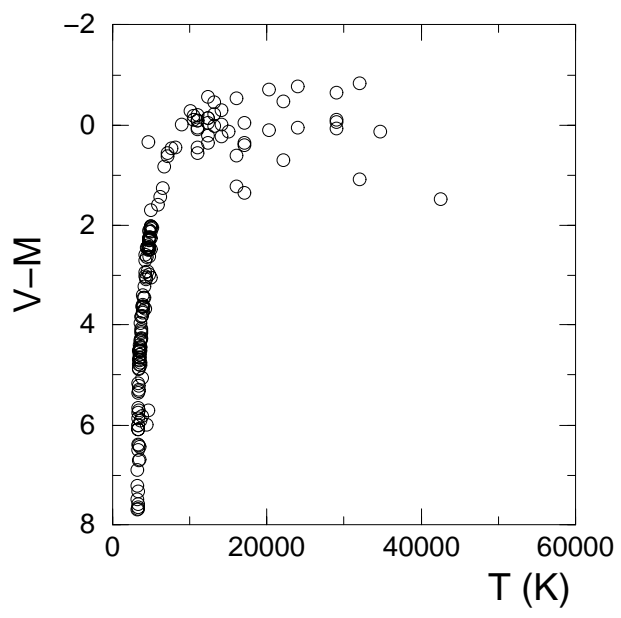

Supergiants

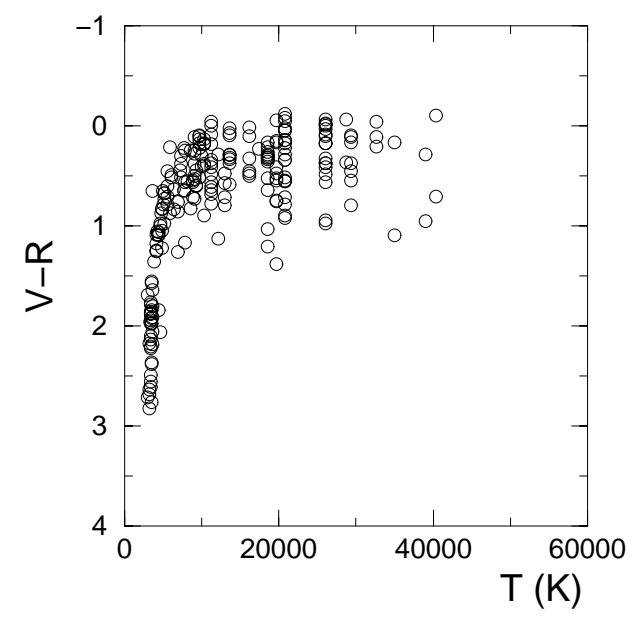

Giants

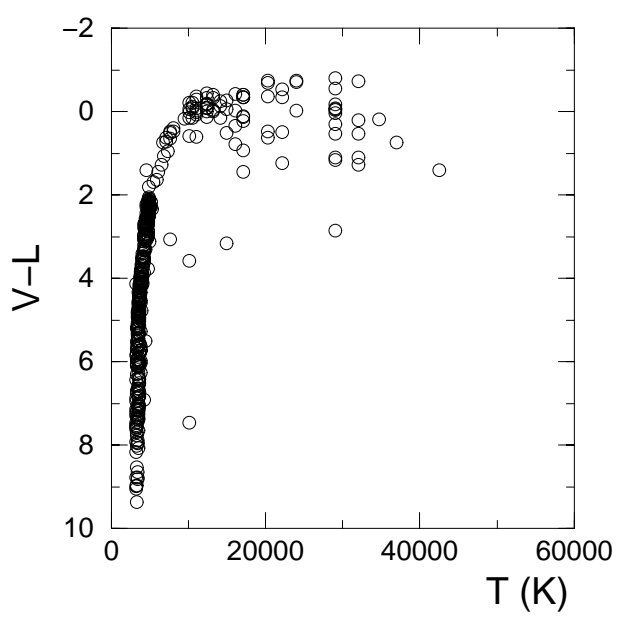

Giants

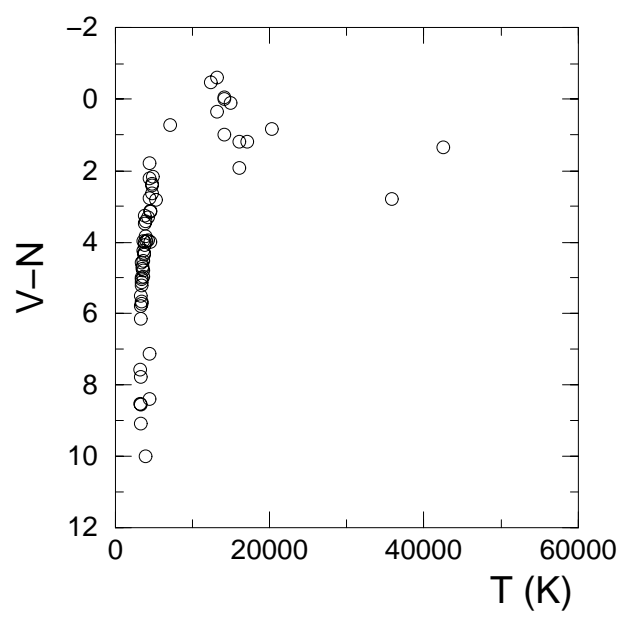

Supergiants

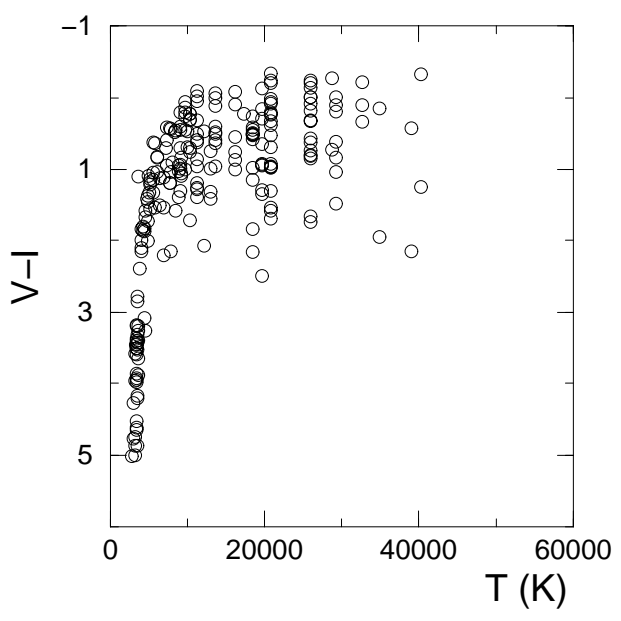

FIG. 6.-Same as Fig. 4, but for giant and supergiant stars 
Supergiants

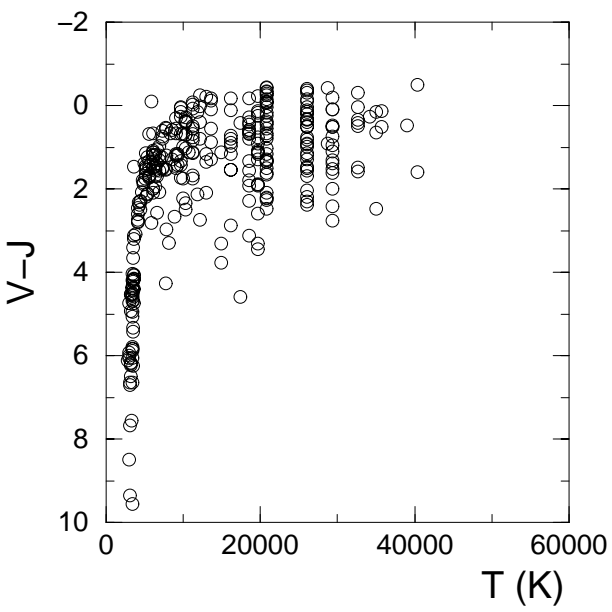

Supergiants

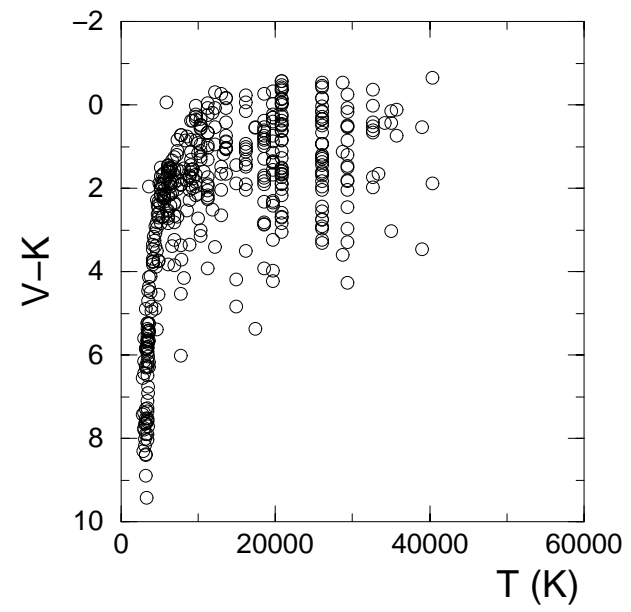

Supergiants

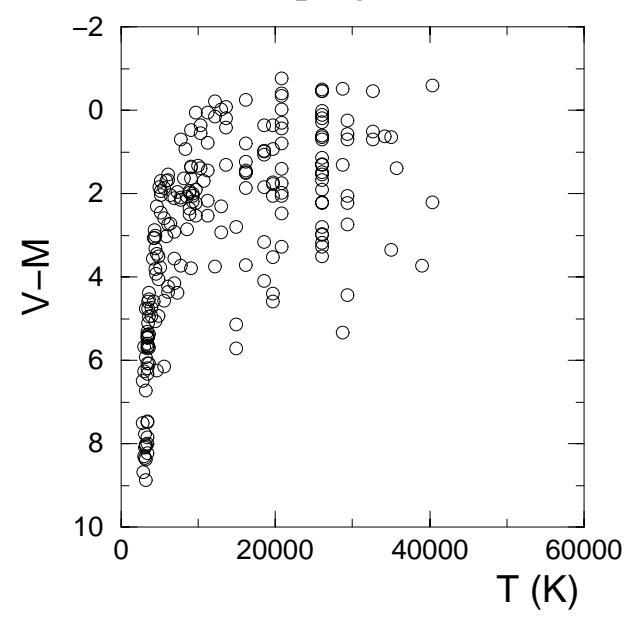

Supergiants

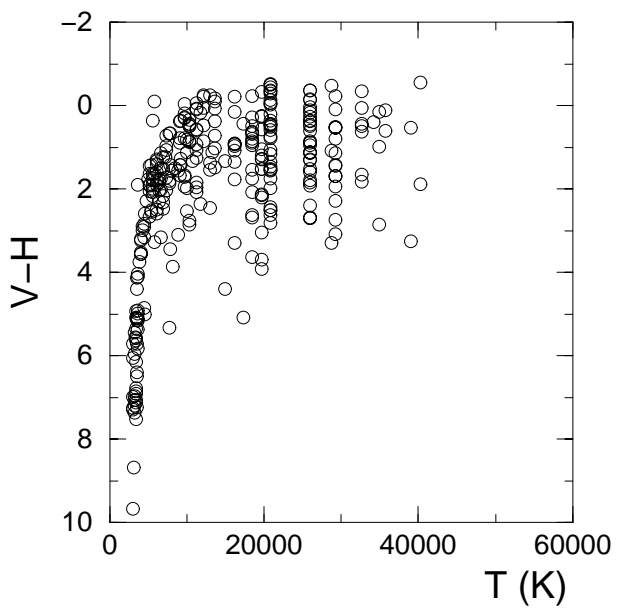

Supergiants

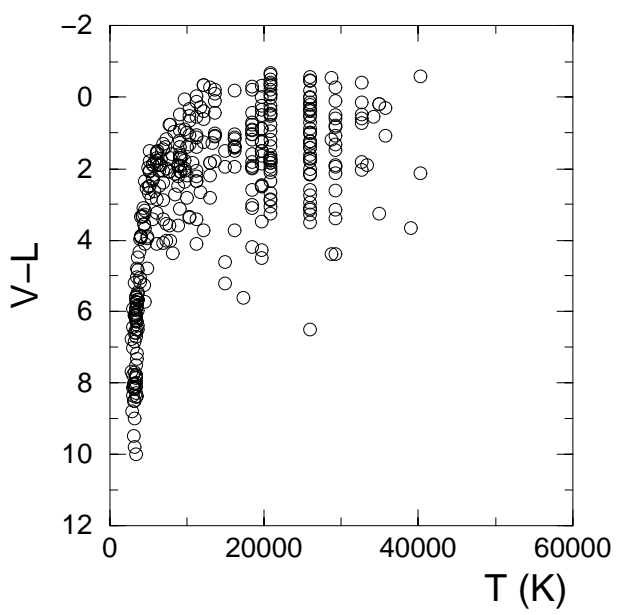

Supergiants

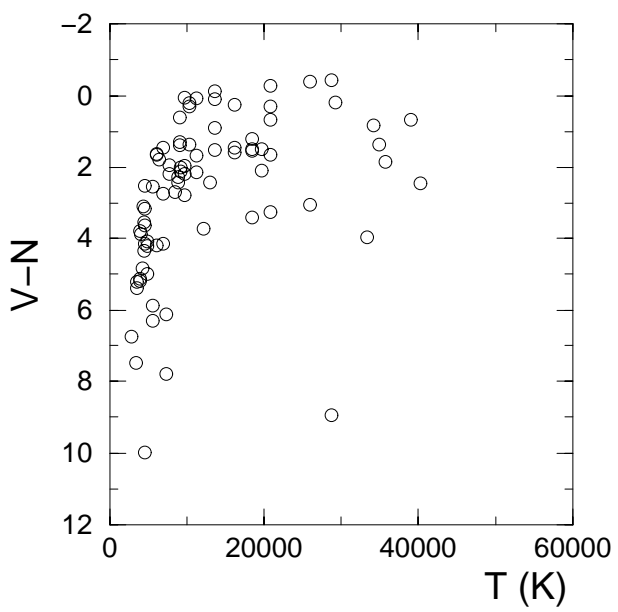

Fig. 7.- Same as Fig. 4, but for supergiant stars 

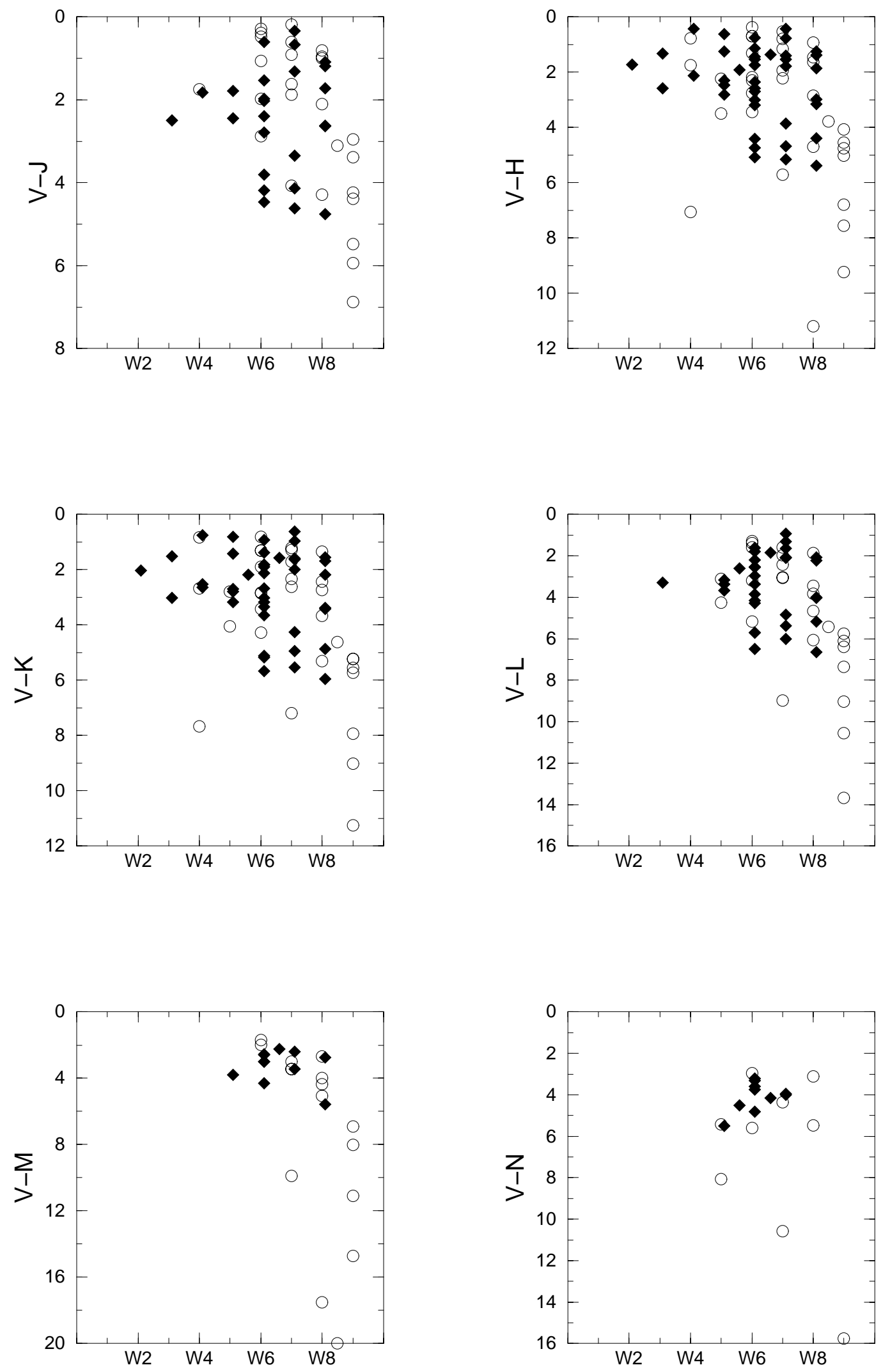

FIG. 8.- Spectral type-color indices for Wolf-Rayet stars. Diamonds denote WN stars; circles denote WC stars. 

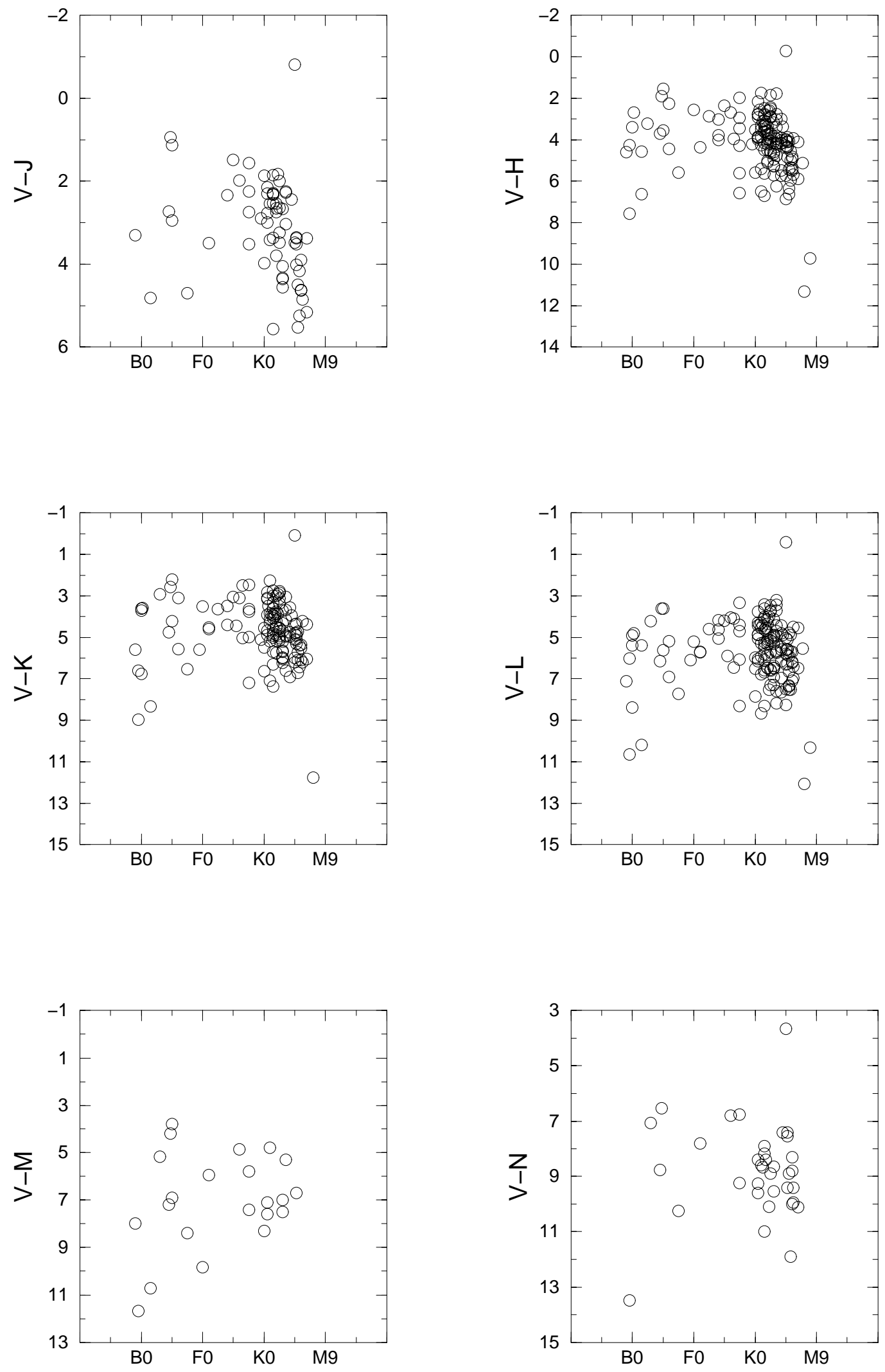

FIG. 9.-Same as Fig. 8, but for T Tauri stars 

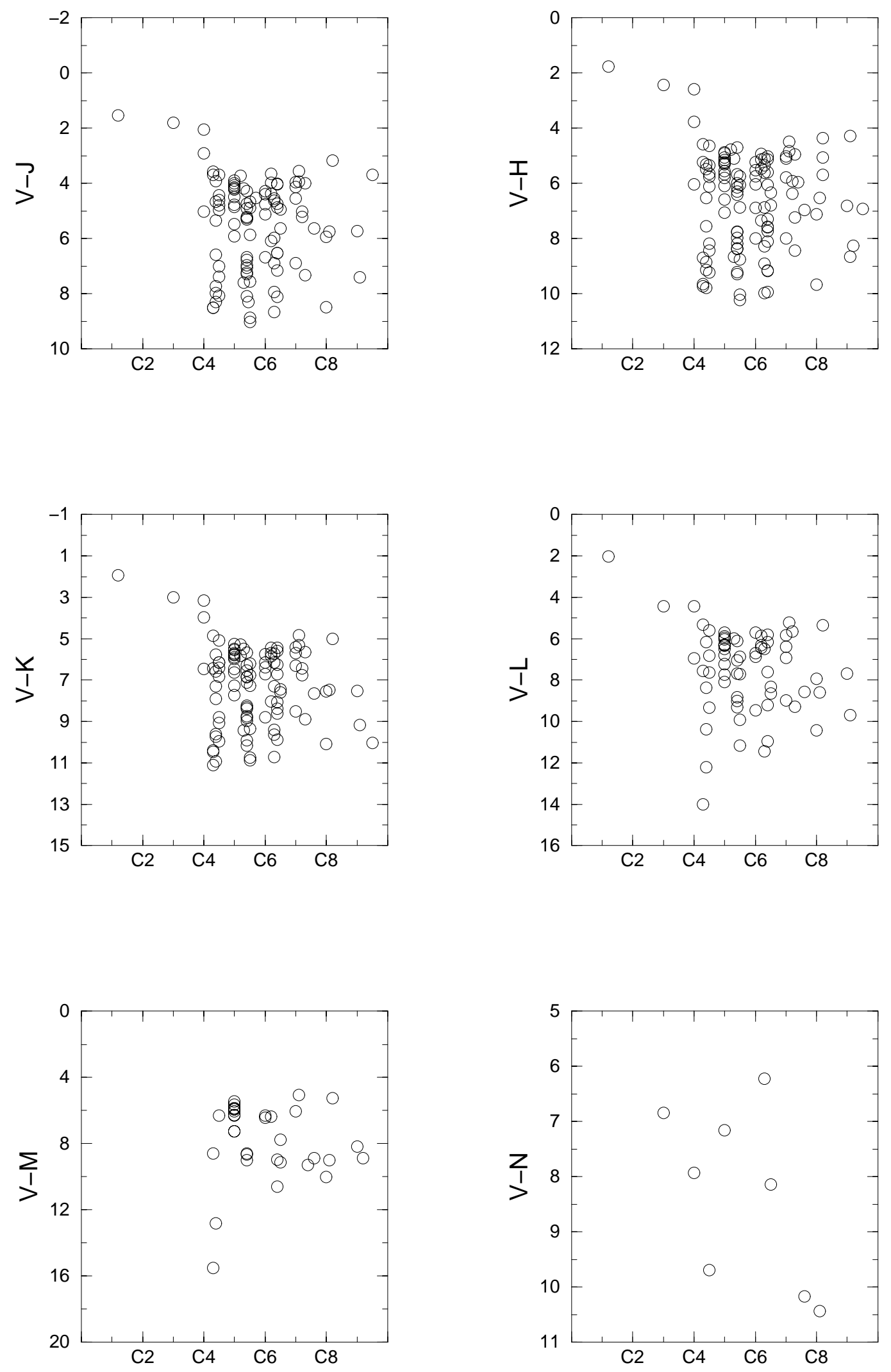

FIG. 10. - Same as Fig. 8, but for carbon stars 
TABLE 2

Number of Stars in EACh Diagram

\begin{tabular}{crrrccc}
\hline \hline Diagram & I & III & V & Carbon & T Tauri & Wolf-Rayet \\
\hline$(V-R)_{0} \ldots \ldots$ & 229 & 408 & 343 & $\ldots$ & $\ldots$ & $\ldots$ \\
$(V-I)_{0} \ldots \ldots$. & 233 & 408 & 345 & $\ldots$ & $\ldots$ & $\ldots$ \\
$(V-J)_{0} \ldots \ldots$. & 349 & 793 & 686 & 122 & 65 & 52 \\
$(V-H)_{0} \ldots \ldots$. & 327 & 514 & 628 & 120 & 148 & 74 \\
$(V-K)_{0} \ldots \ldots$ & 453 & 834 & 758 & 125 & 133 & 75 \\
$(V-L)_{0} \ldots \ldots$. & 381 & 495 & 449 & 85 & 152 & 59 \\
$(V-M)_{0} \ldots \ldots$ & 217 & 180 & 187 & 34 & 22 & 27 \\
$(V-N)_{0} \ldots \ldots$ & 88 & 76 & 57 & 11 & 35 & 19 \\
\hline
\end{tabular}

TABLE 3

Intrinsic Colors of MaIN-Sequence Stars

\begin{tabular}{|c|c|c|c|c|c|c|c|c|}
\hline Spectral Type & $(V-R)_{0}$ & $(V-I)_{0}$ & $(V-J)_{0}$ & $(V-H)_{0}$ & $(V-K)_{0}$ & $(V-L)_{0}$ & $(V-M)_{0}$ & $(V-N)_{0}$ \\
\hline B0 ... & -0.22 & -0.44 & -0.80 & -0.92 & -0.97 & -1.13 & -1.00 & $\ldots$ \\
\hline B0.5 ........ & -0.20 & -0.43 & -0.77 & -0.89 & -0.95 & -1.11 & -0.99 & $\ldots$ \\
\hline B1 $\ldots$ & -0.18 & -0.42 & -0.73 & -0.85 & -0.93 & -1.08 & -0.96 & $\ldots$ \\
\hline B1.5 ......... & -0.17 & -0.41 & -0.70 & -0.82 & -0.91 & -1.05 & -0.94 & $\ldots$ \\
\hline B2 .......... & -0.15 & -0.40 & -0.67 & -0.79 & -0.89 & -1.02 & -0.92 & $\ldots$ \\
\hline B2.5 ........ & -0.14 & -0.39 & -0.64 & -0.76 & -0.86 & -0.97 & -0.88 & -0.96 \\
\hline B3 $\ldots \ldots \ldots \ldots$ & -0.13 & -0.38 & -0.60 & -0.72 & -0.82 & -0.92 & -0.84 & -0.91 \\
\hline B3.5 ........ & -0.12 & -0.37 & -0.58 & -0.70 & -0.80 & -0.90 & -0.82 & -0.87 \\
\hline B4 .......... & -0.11 & -0.35 & -0.56 & -0.68 & -0.77 & -0.86 & -0.79 & -0.84 \\
\hline B4.5 ......... & -0.11 & -0.34 & -0.54 & -0.65 & -0.74 & -0.83 & -0.76 & -0.80 \\
\hline B5 .......... & -0.10 & -0.33 & -0.51 & -0.62 & -0.71 & -0.78 & -0.73 & -0.75 \\
\hline B6 ........... & -0.09 & -0.29 & -0.46 & -0.57 & -0.64 & -0.70 & -0.65 & -0.66 \\
\hline B7 ........... & -0.08 & -0.26 & -0.41 & -0.51 & -0.57 & -0.61 & -0.58 & -0.58 \\
\hline B7.5 ........ & -0.08 & -0.24 & -0.39 & -0.48 & -0.54 & -0.57 & -0.54 & -0.53 \\
\hline B8 $\ldots \ldots \ldots \ldots$ & -0.07 & -0.22 & -0.36 & -0.45 & -0.49 & -0.52 & -0.49 & -0.48 \\
\hline B8.5 .......... & -0.07 & -0.18 & -0.31 & -0.40 & -0.43 & -0.43 & -0.42 & -0.39 \\
\hline B9 .......... & -0.06 & -0.14 & -0.26 & -0.34 & -0.33 & -0.34 & -0.34 & -0.30 \\
\hline B9.5 ........ & -0.03 & -0.11 & -0.22 & -0.29 & -0.26 & -0.27 & -0.26 & -0.22 \\
\hline $\mathrm{A} 0 \ldots \ldots \ldots$ & -0.01 & -0.05 & -0.16 & -0.19 & -0.17 & -0.18 & -0.18 & -0.14 \\
\hline $\mathrm{A} 1 \ldots \ldots \ldots \ldots$ & 0.01 & -0.03 & -0.11 & -0.12 & -0.11 & -0.12 & -0.13 & -0.08 \\
\hline $\mathrm{A} 2 \ldots \ldots \ldots \ldots$ & 0.03 & -0.00 & -0.07 & -0.04 & -0.05 & -0.07 & -0.08 & -0.02 \\
\hline $\mathrm{A} 3 \ldots \ldots \ldots$ & 0.05 & 0.02 & -0.02 & 0.03 & 0.01 & -0.01 & -0.02 & 0.03 \\
\hline A4 .......... & 0.07 & 0.07 & 0.03 & 0.11 & 0.08 & 0.05 & -0.04 & 0.09 \\
\hline A5 .......... & 0.10 & 0.12 & 0.09 & 0.19 & 0.15 & 0.12 & 0.10 & 0.16 \\
\hline A6 ........... & 0.11 & 0.16 & 0.13 & 0.30 & 0.21 & 0.17 & 0.15 & 0.21 \\
\hline A7 ........... & 0.13 & 0.19 & 0.18 & 0.32 & 0.27 & 0.23 & 0.20 & 0.26 \\
\hline A8 $\ldots \ldots \ldots \ldots$ & 0.16 & 0.26 & 0.25 & 0.42 & 0.36 & 0.33 & 0.29 & 0.34 \\
\hline A9 ........... & 0.18 & 0.31 & 0.31 & 0.49 & 0.44 & 0.41 & 0.36 & 0.41 \\
\hline F0 ........... & 0.21 & 0.36 & 0.37 & 0.57 & 0.52 & 0.49 & 0.43 & 0.48 \\
\hline F1 ........... & 0.23 & 0.40 & 0.43 & 0.64 & 0.58 & 0.57 & 0.49 & 0.54 \\
\hline F2 ............ & 0.25 & 0.45 & 0.48 & 0.71 & 0.66 & 0.66 & 0.56 & 0.60 \\
\hline F5 .......... & 0.33 & 0.57 & 0.67 & 0.93 & 0.89 & 0.90 & 0.77 & 0.80 \\
\hline F8 $\ldots \ldots \ldots \ldots$ & 0.37 & 0.64 & 0.79 & 1.06 & 1.03 & 1.06 & 0.91 & 0.91 \\
\hline G0 .......... & 0.41 & 0.70 & 0.87 & 1.15 & 1.14 & 1.18 & 1.01 & 1.01 \\
\hline G2 .......... & 0.45 & 0.75 & 0.97 & 1.25 & 1.26 & 1.31 & 1.12 & 1.11 \\
\hline G3 ........... & 0.45 & 0.76 & 0.98 & 1.27 & 1.28 & 1.33 & 1.14 & 1.13 \\
\hline G5 ........... & 0.47 & 0.78 & 1.02 & 1.31 & 1.32 & 1.38 & 1.18 & 1.17 \\
\hline G8 .......... & 0.52 & 0.85 & 1.14 & 1.44 & 1.47 & 1.55 & 1.34 & 1.30 \\
\hline $\mathrm{K} 0 \ldots \ldots \ldots \ldots$ & 0.61 & 0.97 & 1.34 & 1.67 & 1.74 & 1.85 & 1.61 & 1.54 \\
\hline $\mathrm{K} 1 \ldots \ldots \ldots \ldots$ & 0.67 & 1.05 & 1.46 & 1.80 & 1.89 & 2.02 & 1.78 & 1.68 \\
\hline $\mathrm{K} 2 \ldots \ldots \ldots \ldots$ & 0.73 & 1.14 & 1.60 & 1.94 & 2.06 & 2.21 & 1.97 & 1.84 \\
\hline K3 .......... & 0.80 & 1.25 & 1.73 & 2.09 & 2.23 & 2.40 & 2.17 & 2.01 \\
\hline $\mathrm{K} 4 \ldots \ldots \ldots \ldots$ & 0.86 & 1.34 & 1.84 & 2.22 & 2.38 & 2.57 & 2.36 & 2.15 \\
\hline K5 .......... & 0.97 & 1.54 & 2.04 & 2.46 & 2.66 & 2.87 & 2.71 & 2.44 \\
\hline K7 ........... & 1.13 & 1.86 & 2.30 & 2.78 & 3.01 & 3.25 & 3.21 & 2.83 \\
\hline M0.......... & 1.26 & 2.15 & 2.49 & 3.04 & 3.29 & 3.54 & 3.65 & 3.16 \\
\hline M1.......... & 1.36 & 2.36 & 2.61 & 3.22 & 3.47 & 3.72 & 3.95 & 3.39 \\
\hline M2 ........... & 1.46 & 2.62 & 2.74 & 3.42 & 3.67 & 3.92 & 4.31 & 3.66 \\
\hline M3.......... & 1.56 & 2.84 & 2.84 & 3.58 & 3.83 & 4.08 & 4.62 & 3.89 \\
\hline M4 .......... & 1.65 & 3.07 & 2.93 & 3.74 & 3.98 & 4.22 & 4.93 & 4.11 \\
\hline
\end{tabular}


TABLE 4

INTRINSIC COLORS OF Giant StaRs

\begin{tabular}{|c|c|c|c|c|c|c|c|c|}
\hline Spectral Type & $(V-R)_{0}$ & $(V-I)_{0}$ & $(V-J)_{0}$ & $(V-H)_{0}$ & $(V-K)_{0}$ & $(V-L)_{0}$ & $(V-M)_{0}$ & $(V-N)_{0}$ \\
\hline G3 $\ldots \ldots \ldots \ldots$ & 0.43 & 0.75 & 0.83 & 1.32 & 1.36 & 1.44 & 1.38 & 1.37 \\
\hline G3.5 $\ldots \ldots \ldots \ldots$ & 0.44 & 0.77 & 0.86 & 1.35 & 1.40 & 1.47 & 1.42 & 1.40 \\
\hline G4 ............ & 0.45 & 0.79 & 0.89 & 1.38 & 1.45 & 1.51 & 1.46 & 1.44 \\
\hline G5 ........... & 0.48 & 0.83 & 0.94 & 1.44 & 1.53 & 1.58 & 1.53 & 1.51 \\
\hline G8 $\ldots \ldots \ldots \ldots$ & 0.55 & 0.95 & 1.11 & 1.61 & 1.77 & 1.79 & 1.73 & 1.75 \\
\hline G9 ............ & 0.57 & 0.99 & 1.17 & 1.66 & 1.85 & 1.88 & 1.82 & 1.83 \\
\hline G9.5 .......... & 0.58 & 1.01 & 1.20 & 1.69 & 1.90 & 1.92 & 1.86 & 1.88 \\
\hline K0 ............ & 0.60 & 1.03 & 1.23 & 1.72 & 1.94 & 1.97 & 1.90 & 1.92 \\
\hline $\mathrm{K} 0.5 \ldots \ldots \ldots$ & 0.63 & 1.07 & 1.30 & 1.79 & 2.03 & 2.07 & 2.00 & 2.02 \\
\hline $\mathrm{K} 1 \ldots \ldots \ldots \ldots$ & 0.66 & 1.12 & 1.37 & 1.87 & 2.12 & 2.17 & 2.09 & 2.11 \\
\hline $\mathrm{K} 2 \ldots \ldots \ldots \ldots$ & 0.74 & 1.23 & 1.56 & 2.08 & 2.36 & 2.44 & 2.35 & 2.37 \\
\hline $\mathrm{K} 2.5 \ldots \ldots \ldots$ & 0.80 & 1.30 & 1.69 & 2.23 & 2.52 & 2.62 & 2.52 & 2.54 \\
\hline K3 ............ & 0.86 & 1.39 & 1.84 & 2.40 & 2.69 & 2.82 & 2.70 & 2.73 \\
\hline K3.5 ......... & 0.91 & 1.49 & 1.99 & 2.73 & 2.86 & 3.01 & 2.88 & 2.91 \\
\hline K4 ............ & 0.96 & 1.61 & 2.16 & 2.77 & 3.05 & 3.22 & 3.08 & 3.02 \\
\hline K5 ............ & 0.99 & 1.67 & 2.25 & 2.87 & 3.14 & 3.33 & 3.18 & 3.21 \\
\hline $\mathrm{M} 0 \ldots \ldots \ldots \ldots$ & 1.09 & 1.91 & 2.55 & 3.23 & 3.46 & 3.69 & 3.51 & 3.54 \\
\hline $\mathrm{M} 0.5 \ldots \ldots \ldots$ & 1.13 & 1.98 & 2.64 & 3.33 & 3.55 & 3.79 & 3.61 & 3.64 \\
\hline $\mathrm{M} 1 \ldots \ldots \ldots \ldots$ & 1.16 & 2.07 & 2.73 & 3.45 & 3.64 & 3.90 & 3.70 & 3.74 \\
\hline $\mathrm{M} 2 \ldots \ldots \ldots \ldots$ & 1.27 & 2.32 & 2.99 & 3.76 & 3.89 & 4.18 & 3.96 & 4.00 \\
\hline 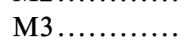 & 1.39 & 2.61 & 3.25 & 4.08 & 4.13 & 4.46 & 4.22 & 4.26 \\
\hline $\mathrm{M} 4 \ldots \ldots \ldots \ldots$ & 1.58 & 3.04 & 3.58 & 4.49 & 4.42 & 4.79 & 4.52 & 4.56 \\
\hline $\mathrm{M} 4.5 \ldots \ldots \ldots$ & 1.70 & 3.32 & 3.76 & 4.73 & 4.57 & 4.97 & 4.69 & 4.73 \\
\hline M5 ........... & 1.85 & 3.67 & 3.95 & 4.98 & 4.73 & 5.15 & 4.86 & 4.90 \\
\hline M6 ........... & 2.24 & 4.53 & 4.34 & 5.50 & 5.04 & 5.51 & 5.18 & 5.23 \\
\hline
\end{tabular}


TABLE 5

INTRINSIC COLORS OF SUPERgIANT StaRS

\begin{tabular}{|c|c|c|c|c|c|c|c|c|}
\hline Spectral Type & $(V-R)_{0}$ & $(V-I)_{0}$ & $(V-J)_{0}$ & $(V-H)_{0}$ & $(V-K)_{0}$ & $(V-L)_{0}$ & $(V-M)_{0}$ & $(V-N)_{0}$ \\
\hline O9. & -0.19 & -0.41 & -0.57 & -0.75 & -0.84 & -0.78 & -0.99 & -0.65 \\
\hline O9.5 .......... & -0.18 & -0.39 & -0.55 & -0.73 & -0.82 & -0.75 & -0.95 & -0.63 \\
\hline B0 ............. & -0.17 & -0.37 & -0.53 & -0.70 & -0.78 & -0.71 & -0.90 & -0.59 \\
\hline B $0.5 \ldots \ldots \ldots$ & -0.16 & -0.35 & -0.50 & -0.66 & -0.75 & -0.67 & -0.84 & -0.55 \\
\hline B1 .......... & -0.15 & -0.32 & -0.47 & -0.62 & -0.70 & -0.61 & -0.77 & -0.50 \\
\hline B1.5 .......... & -0.14 & -0.30 & -0.45 & -0.59 & -0.67 & -0.58 & -0.73 & -0.47 \\
\hline B2 $\ldots \ldots \ldots \ldots$ & -0.14 & -0.29 & -0.43 & -0.56 & -0.63 & -0.55 & -0.68 & -0.44 \\
\hline B3 $\ldots \ldots \ldots \ldots$ & -0.11 & -0.25 & -0.37 & -0.49 & -0.54 & -0.45 & -0.57 & -0.35 \\
\hline B4 ............. & -0.09 & -0.22 & -0.33 & -0.42 & -0.48 & -0.38 & -0.48 & -0.28 \\
\hline B5 $\ldots \ldots \ldots \ldots$ & -0.07 & -0.19 & -0.28 & -0.34 & -0.39 & -0.30 & -0.38 & -0.19 \\
\hline B6 ............ & -0.06 & -0.17 & -0.24 & -0.30 & -0.34 & -0.25 & -0.32 & -0.14 \\
\hline B7 ............ & -0.04 & -0.14 & -0.20 & -0.24 & -0.27 & -0.18 & -0.24 & -0.06 \\
\hline B7.5 .......... & -0.03 & -0.13 & -0.17 & -0.20 & -0.22 & -0.14 & -0.19 & -0.02 \\
\hline B8 $\ldots \ldots \ldots \ldots$ & -0.01 & -0.10 & -0.12 & -0.14 & -0.15 & -0.07 & -0.11 & 0.05 \\
\hline B9 ............. & 0.02 & -0.03 & -0.04 & -0.03 & -0.02 & 0.06 & 0.03 & 0.19 \\
\hline B9.5 ........ & 0.04 & 0.00 & -0.01 & 0.01 & 0.03 & 0.11 & 0.08 & 0.23 \\
\hline A0 .......... & 0.05 & 0.03 & 0.02 & 0.06 & 0.08 & 0.15 & 0.13 & 0.29 \\
\hline $\mathrm{A} 1 \ldots \ldots \ldots \ldots$ & 0.08 & 0.08 & 0.09 & 0.14 & 0.18 & 0.25 & 0.23 & 0.39 \\
\hline $\mathrm{A} 2 \ldots \ldots \ldots \ldots$ & 0.09 & 0.10 & 0.11 & 0.17 & 0.21 & 0.28 & 0.27 & 0.43 \\
\hline A5 $\ldots \ldots \ldots \ldots$ & 0.13 & 0.18 & 0.20 & 0.29 & 0.35 & 0.41 & 0.41 & 0.57 \\
\hline F0 ............ & 0.20 & 0.31 & 0.36 & 0.51 & 0.60 & 0.64 & 0.65 & 0.82 \\
\hline $\mathrm{F} 2 \ldots \ldots \ldots \ldots$ & 0.24 & 0.37 & 0.44 & 0.62 & 0.73 & 0.77 & 0.77 & 0.95 \\
\hline F5 ........... & 0.29 & 0.47 & 0.57 & 0.79 & 0.91 & 0.95 & 0.96 & 1.15 \\
\hline F8 $\ldots \ldots \ldots \ldots$ & 0.43 & 0.70 & 0.87 & 1.17 & 1.34 & 1.35 & 1.36 & 1.58 \\
\hline G0 $\ldots \ldots \ldots \ldots$ & 0.55 & 0.90 & 1.14 & 1.52 & 1.71 & 1.72 & 1.72 & 1.98 \\
\hline $\mathrm{G} 2 \ldots \ldots \ldots \ldots$ & 0.65 & 1.06 & 1.35 & 1.80 & 1.99 & 2.01 & 1.99 & 2.28 \\
\hline G3 ........... & 0.68 & 1.12 & 1.43 & 1.90 & 2.09 & 2.11 & 2.09 & 2.38 \\
\hline $\mathrm{G} 3.5 \ldots \ldots \ldots$ & 0.70 & 1.16 & 1.47 & 1.95 & 2.15 & 2.17 & 2.14 & 2.44 \\
\hline $\mathrm{G} 4 \ldots \ldots \ldots \ldots$ & 0.72 & 1.19 & 1.52 & 2.01 & 2.20 & 2.23 & 2.20 & 2.50 \\
\hline G5 ........... & 0.77 & 1.26 & 1.61 & 2.13 & 2.32 & 2.35 & 2.31 & 2.63 \\
\hline G8 ........... & 0.87 & 1.43 & 1.83 & 2.41 & 2.59 & 2.64 & 2.58 & 2.91 \\
\hline K0 ............ & 0.95 & 1.59 & 2.01 & 2.64 & 2.80 & 2.87 & 2.79 & 3.14 \\
\hline $\mathrm{K} 1 \ldots \ldots \ldots \ldots$ & 0.99 & 1.68 & 2.11 & 2.76 & 2.91 & 3.00 & 2.90 & 3.26 \\
\hline $\mathrm{K} 2 \ldots \ldots \ldots \ldots$ & 1.03 & 1.76 & 2.20 & 2.87 & 3.01 & 3.12 & 3.01 & 3.38 \\
\hline K3 $3 \ldots \ldots \ldots$ & 1.13 & 1.96 & 2.41 & 3.14 & 3.25 & 3.39 & 3.25 & 3.63 \\
\hline K3.5 .......... & 1.16 & 2.04 & 2.50 & 3.25 & 3.34 & 3.50 & 3.35 & 3.74 \\
\hline K4 ............ & 1.20 & 2.13 & 2.59 & 3.37 & 3.44 & 3.62 & 3.46 & 3.84 \\
\hline K5 $\ldots \ldots \ldots \ldots$ & 1.27 & 2.27 & 2.74 & 3.55 & 3.59 & 3.80 & 3.62 & 4.01 \\
\hline M0.......... & 1.42 & 2.59 & 3.07 & 3.97 & 3.92 & 4.22 & 3.99 & 4.38 \\
\hline M0.5 ......... & 1.46 & 2.68 & 3.16 & 4.08 & 4.01 & 4.33 & 4.09 & 4.48 \\
\hline M1 .................. & 1.50 & 2.77 & 3.25 & 4.20 & 4.10 & 4.45 & 4.19 & 4.58 \\
\hline $\mathrm{M} 1.5 \ldots \ldots \ldots \ldots$ & 1.54 & 2.87 & 3.35 & 4.32 & 4.19 & 4.57 & 4.29 & 4.69 \\
\hline $\mathrm{M} 2 \ldots \ldots \ldots \ldots$ & 1.58 & 2.97 & 3.45 & 4.45 & 4.28 & 4.70 & 4.40 & 4.80 \\
\hline $\mathrm{M} 2.5 \ldots \ldots \ldots$ & 1.70 & 3.24 & 3.73 & 4.79 & 4.53 & 5.04 & 4.69 & 5.07 \\
\hline M3............ & 1.83 & 3.55 & 4.03 & 5.16 & 4.79 & 5.41 & 5.01 & 5.37 \\
\hline $\mathrm{M} 3.5 \ldots \ldots \ldots$ & 1.95 & 3.86 & 4.32 & 5.53 & 5.03 & 5.77 & 5.31 & 5.65 \\
\hline M4 ............ & 2.09 & 4.20 & 4.65 & 5.93 & 5.29 & 6.17 & 5.64 & 5.95 \\
\hline M5........... & 2.34 & 4.85 & 5.26 & 6.68 & 5.73 & 6.90 & 6.25 & 6.47 \\
\hline
\end{tabular}

TABLE 6

INTRINSIC COLORS OF Wolf-RAYet, T TAURI, AND CARbon Stars

\begin{tabular}{ccccccc}
\hline \hline Star Type & $(V-J)_{0}$ & $(V-H)_{0}$ & $(V-K)_{0}$ & $(V-L)_{0}$ & $(V-M)_{0}$ & $(V-N)_{0}$ \\
\hline WN, WC .............. & $<0.0$ & $<0.0$ & $<0.5$ & $<1.0$ & $<1.5$ & $<3.0$ \\
T Tauri ............... & $<1.0$ & $<1.0$ & $<2.0$ & $<3.0$ & $<4.0$ & $<6.0$ \\
Carbon (C4 to C9)..... & 3.0 & 4.0 & 5.0 & 5.0 & 5.0 & $<6.0$ \\
\hline
\end{tabular}


affected by interstellar reddening. The colors of $\mathrm{T}$ Tauri stars are very stable with respect to spectral class. This can be because extinction is stronger for the few blue $T$ Tauri stars, which are, in general, embedded in clouds of dust and gas than for late $\mathrm{T}$ Tauri stars. The exception seems to be in the $(V-J)$ color, which is, in the near-infrared, more affected by interstellar extinction than the colors from longer wavelengths.

Table 2 presents the number of stars used in each diagram. Tables $3,4,5$, and 6 give the intrinsic colors. It should be noted that, for the infrared, $V-\lambda$ departs from the zero-point criteria used by Johnson in the calibration in $U B V$, where all colors for $\mathrm{A} 0$ stars are set to zero. This was already observed by Johnson himself (Johnson 1966) for A0 supergiants, where $V-K=0.10$, and even for the main sequence, where $V-K=-0.03$. Koornneef (1983b) gives $V-K=0.21$ for $\mathrm{A} 0$ supergiants and 0.00 for the main sequence. In this sense, it is not surprising that in the present paper we find that $V-K=0.08$ for supergiant stars and -0.17 for main-sequence stars. In fact, our results were derived, for the $V-K$ color, from data where $15 \mathrm{~A} 0$ mainsequence stars, from a total sample of $36 \mathrm{~A} 0$ stars, are bluer than 0.00 . The curve that defines the zero-reddening envelope has the value -0.17 at the point corresponding to
A0 stars; we note that three stars have $V-K$ even bluer than the -0.17 value we adopted as the intrinsic color. We note also that the difference between $V-K=0.00$, the value expected by a zero-point calibration, and $V-K=-0.17$, the one derived in this paper, is far larger than the expected error in the $K$ photometry, as shown in Table 1. We therefore conclude that $(V-K)$-values for A0 stars that differ from 0.00 are not only real, but are not even new in the literature.

We note that, for dwarfs and giants, our indices are in general bluer than those previously published. This is easily understood, because Johnson's tables, being obtained from averages, tend to produce redder colors. For supergiants, differences are smaller and, although systematic for stars hotter than F, fall within uncertainties for infrared photometry as listed in Table 1 . The reason for this bias perhaps has its origin in the calibration used by Kron (1958); however, we must have in mind that our sample is far larger than Johnson's.

This work benefited from data extracted from SIMBAD database, Strasbourg Data Center, France. Scholarships from Fapergs and UFRGS are acknowledged.
Allen, C. W. 1973, Astrophysical Quantities (London: Athlone Press)

Allen, D. A., \& Cragg, T. A. 1983, MNRAS, 203, 777

Bessell, M. S., \& Brett, J. M. 1988, PASP, 100, 1134

Bouchet, P., Manfroid, J., \& Schmider, F. X. 1991, A\&AS, 91, 409

Carter, B. S. 1990, MNRAS, 242, 1

Catchpole, R. M., Robertson, B. S. C., Lloyd-Evans, T. H. H., Feast, M. W., Glass, I. S., \& Carter, B. S. 1979, South African Astron. Obs. Circ., 1, 61 Cohen, M. 1974, MNRAS, 169, 257

Ducati, J. R. 1993, Wisconsin Astrophysics, 504

Engels, D., Sherwood, W. A., Wamsteker, W., \& Schultz, G. V. 1981, A\&AS, 45,5

Frogel, J. A., Persson, S. E., Aaronson, M., \& Matthews, K. 1978, ApJ, 220, 75

Gehrz, R. D., Hackwell, J. A., \& Jones, T. W. 1974, ApJ, 191, 675

Gehrz, R. D., \& Woolf, N. J. 1971, ApJ, 165, 285

Gezari, D. Y., Schmitz, M., Pitts, P. S., \& Mead, J. M. 1993, Catalog of Infrared Observations (NASA RP-1294) (3d ed.; Washington: NASA)

Gillett, F. C., Merrill, K. M., \& Stein, W. A. 1971, ApJ, 164, 83

Glass, I. S. 1974, Mon. Notes Astron. Soc. South Africa, 33, 53

Hoffleit, D., \& Jaschek, C. 1982, The Bright Star Catalogue (4th rev. ed.; New Haven: Yale Univ. Obs.)

Hoffleit, D., Saladyga, M., \& Wlasuk, P. 1983, A Supplement to the Bright Star Catalogue (New Haven: Yale Univ. Obs.)

Jaschek, C., \& Jaschek, M. 1987, The Classification of Stars (Cambridge: Cambridge Univ. Press)

Johnson, H. L. 1966, ARA\&A, 4, 193

Johnson, H. L., Iriarte, B., Mitchell, R. I., \& Wisniewski, W. Z. 1966, Comm. Lunar Planet. Lab., 4, 99

\section{REFERENCES}

Kaler, J. B. 1989, Stars and Their Spectra (Cambridge: Cambridge Univ. Press)

Koornneef, J. 1983a, A\&AS, 51, 489

.1983b, A\&A, 128, 84

Kron, G. E. 1958, PASP, 70, 561

Lang, K. R. 1992, Astrophysical Data: Planets and Stars (New York: Springer)

Lanz, T. 1986, A\&AS, 65, 195

Lee, T. A. 1970, ApJ, 162, 217

Mihalas, D., \& Binney, J. 1981, Galactic Astronomy: Structure and Kinematics (2d ed.; San Francisco: Freeman)

Philip, A. G. D., \& Egret, D. 1980, A\&AS, 40, 199

Rydgren, A. E., Strom, S. E., \& Strom, K. M. 1976, ApJS, 30, 307

Schmidt-Kaler, T. H. 1982, in Landolt-Börnstein, New Series, Group VI, Vol. 2b, Stars and Star Clusters, ed. K. Schaifers \& H. H. Voigt (New York: Springer)

Selby, M. J., Hepburn, I., Blackwell, D. E., Booth, A. J., Haddock, D. J., Arribas, S., Leggett, S. K., \& Mountain, C. M. 1988, A\&AS, 74, 127

Sinton, W. M., \& Tittemore, W. C. 1984, AJ, 89, 1366

Sterken, C., \& de Loore, C. W. H. 1982, in IAU Symp. 99, Wolf-Rayet Stars: Observations, Physics, Evolution, ed. C. W. H. de Loore \& A. J. Willis (Dordrecht: Reidel), 67

Thé, P. S., Wesselius, P. R., \& Janssen, I. M. H. H. 1986, A\&AS, 66, 63

Tokunaga, A. T. 1984, AJ, 89, 172

Wegner, W. 1994, MNRAS, 270, 229

Whittet, D. C. B., \& van Breda, I. G. 1980, MNRAS, 192, 467 